$56 \mid 2008$

Le film d'Art \& les films d'art en Europe (1908-1911)

\title{
Au-delà du film d'art. Sur deux films retrouvés à la Cinémathèque de Toulouse
}

Beyond film d'art. On two films rediscovered at the Cinémathèque de Toulouse

\section{Christophe Gauthier}

\section{OpenEdition}

\section{Journals}

Édition électronique

URL : https://journals.openedition.org/1895/4083

DOI : $10.4000 / 1895.4083$

ISBN : 978-2-8218-0990-1

ISSN : 1960-6176

Éditeur

Association française de recherche sur l'histoire du cinéma (AFRHC)

Édition imprimée

Date de publication : 1 décembre 2008

Pagination : 337-334

ISBN : 978-2-913758-57-5

ISSN : 0769-0959

Référence électronique

Christophe Gauthier, «Au-delà du film d'art. Sur deux films retrouvés à la Cinémathèque de Toulouse », 1895. Mille huit cent quatre-vingt-quinze [En ligne], 56 | 2008, mis en ligne le 01 décembre 2011, consulté le 16 avril 2022. URL : http://journals.openedition.org/1895/4083 ; DOI : https://doi.org/ $10.4000 / 1895.4083$ 


\title{
Au-delà du film d'art Sur deux films retrouvés à la Cinémathèque de Toulouse
}

\author{
par Christophe Gauthier
}

\section{Prologue}

Dans les années 1970, Raymond Borde fit entrer dans les collections de la Cinémathèque de Toulouse un ensemble de films nitrate des années 1909-1915, issus entre autres des séries d'art produites par Pathé. On y recense notamment la Vengeance du sire de Guildo de Gérard Bourgeois (1909), Fouquet, I'homme au masque de fer de Camille de Morlhon (1910) ou encore le Siège de Calais d'Henri Andréani (1910). À une époque où le plan nitrate n'existait pas encore, Borde avait expédié ces copies à la cinémathèque nationale de Roumanie, afin

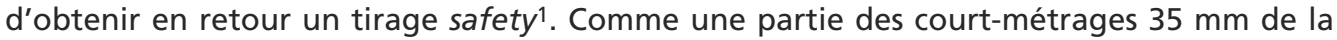
collection, le catalogage de ces films avait été effectué rapidement, aux fins (essentielles) d'une identification a minima et d'une localisation précise dans les magasins. Grâce à l'ouverture du Centre de conservation et de recherche de la Cinémathèque de Toulouse en 2004, le catalogue des fonds anciens de la collection, à commencer par les films muets, a été progressivement repris. Dans ce même stock figuraient donc un Macbeth ${ }^{2}$ et un Pelléas et Mélisande ${ }^{3}$ que ces récents travaux ont permis d'identifier. Réalisés par un certain Gustave

1 Cette pratique courante à la Cinémathèque de Toulouse - qui ne disposait pas de tireuse ni de développeuse - concernait les films sur support nitrate et les films sur support safety tirés dans les années 1920 (support diacétate). Elle a été marquée par une intense collaboration avec la Cinémathèque royale de Belgique, alors dirigée par Jacques Ledoux, et en second lieu avec l'Archive nationale du film de Bucarest. Elle s'est poursuivie jusqu'à l'institution en 1991 du « plan nitrate » ou plan national de sauvegarde des films anciens qui a entraîné le dépôt des films nitrate de la collection aux Archives françaises du film du CNC. 2 Copie $35 \mathrm{~mm} \mathrm{n}^{\circ}$ 8560-CM de la Cinémathèque de Toulouse, NB, Muet, INTFR, $906 \mathrm{~m}$. 3 Copie $35 \mathrm{~mm} \mathrm{n}{ }^{\circ} 8514-\mathrm{CM}$ de la Cinémathèque de Toulouse, NB, Muet, INTFR, $489 \mathrm{~m}$. Le tirage très médiocre, voire défectueux à certains endroits, de ces deux copies n'en permet malheureusement pas la projection. 
Labruyère, opérateur de la firme Éclair en activité dans les années 1900 et 1910, ils ont pour interprètes principaux Georgette Leblanc-Maeterlinck ${ }^{4}$ et Séverin-Mars ${ }^{5}$ - dont ce sont pour ainsi dire les premières apparitions à l'écran ${ }^{6}$ - et sont tournés dans les décors naturels offerts par l'abbaye de Saint-Wandrille. Tout semblait simple jusqu'à ce que quelques recherches nous amènent à reconsidérer I'histoire de ces deux films, a priori disparus en dehors de la Cinémathèque de Toulouse - et donc de celle de Bucarest - une histoire en réalité bien plus complexe qu'il n'y paraît, et qui laisse clairement entrevoir que si Gustave Labruyère était bien derrière la caméra, il ne fut nullement le metteur en scène de ces deux films.

\section{Acte I : Abbaye de Saint-Wandrille, été 1908}

Le 30 novembre 1907, Maurice Maeterlinck est devenu locataire pour un bail de dix-huit ans de l'abbaye de Saint-Wandrille dont les derniers moines ont été expulsés en 1901 en raison de l'interdiction des Congrégations7. Georgette Leblanc et l'auteur de Pelléas y avaient passé leur premier été en 19078. Le couple s'installe dans le corps de logis des XVII et XVIII siècles (infirmerie et hôtellerie) et transforme le réfectoire en salle à manger. II y séjourne régulièrement jusqu'en 1919. L'aménagement de l'abbaye (une demi-ruine, dont tout confort est banni) est laissé à l'appréciation de Georgette Leblanc qui repeint toutes les pièces et les meuble de tables gigantesques, de chaises trouvées sur place, de grands coffres Renaissance. Ses Souvenirs parus en 1931 laissent entrevoir le bonheur qu'elle eut à occuper ces lieux, à s'y promener sans fin (" Immobile, retenant mon souffle, je sentais mon corps devenir une chose parmi les choses ${ }^{9}$ ) tandis que le poète - pas encore « nobélisé $»-$ se délasse en traversant

4 Georgette Leblanc (1869-1941) est comédienne mais aussi cantatrice. Proche de Mallarmé et du milieu symboliste à la fin du XIXe siècle, elle est au moment du tournage du film l'épouse de Maurice Maeterlinck, I'auteur de l'Oiseau bleu et de Pelléas et Mélisande, et dont elle se séparera définitivement après la guerre, Elle est aussi la sœur de l'écrivain Maurice Leblanc. Sur la vie de Georgette Leblanc, voir Maxime Benoît-Jannin, Georgette Leblanc (1869-1941). Biographie, Bruxelles, Le Cri, 1998. 5 Issu du théâtre, Armand-Jean de Malafayde, dit Séverin-Mars (1873-1921) est l'interprète principal de la Dixième symphonie (1917), J'Accuse (1919) et la Roue (1923) d'Abel Gance. En 1921, Il réalise également pour le cinéma le Cœur magnifique d'après une de ses propres œuvres. 6 Première apparition effectivement pour Georgette Leblanc, et la seule avant I'Inhumaine en 1923, malgré de nombreuses tentatives entre 1915 et 1923 et une contribution remarquée à la réflexion esthétique sur le cinéma dans un article du Mercure de France en 1919. Sur Georgette Leblanc et le cinéma, nous renvoyons à François Albera, notice "Georgette Leblanc », dans F. Albera et J. A. Gili (dir.), Dictionnaire du cinéma français des années vingt, 1895, juin 2001, n³3, p. 244-246. Séverin-Mars avait en revanche déjà tourné dans quelques films dont le Duel d'un fou réalisé en 1913 par Andreyor (copie 35 mm n 763-CM de la Cinémathèque de Toulouse, NB, Muet, INTFR, 510 m). 7 Située en Haute-Normandie, non loin de la propriété familiale des Leblanc, l'abbaye est à nouveau occupée par les moines à partir de 1931. 8 Le couple partageait ses hivers entre la villa " les Abeilles " dans les environs de Grasse et leur appartement de Neuilly. 9 G. Leblanc, Souvenirs (1895-1918), Paris, Grasset, 1931, p. 232. 
salle du chapitre et réfectoire en patins à roulettes (" II roulait à toute vitesse, la pipe à la bouche, un livre sous le bras et son chien sur les talons $\left.{ }^{10}\right)$.

L'espace est ainsi habité le temps de quelques étés par une comédienne et un auteur qui sont alors parmi les plus grandes célébrités de leur époque. Ils y reçoivent personnalités du monde littéraire et politique. Georgette Leblanc, séduite par le lieu et par les possibilités dramatiques qu'il semble offrir, souhaite très vite y monter un spectacle, en décors naturels, mais non à l'imitation du théâtre en plein air qu'elle voit comme un " camouflage »11 de la nature. Le premier projet ne concerne pas une pièce de Maeterlinck mais le Macbeth de Shakespeare, traduit et adapté par le poète ${ }^{12}$. Georgette Leblanc explique les raisons de ce choix :

On ignore quel surcroît d'émotions peuvent donner l'art et la nature mêlés - une phrase de Shakespeare rendue à la terre, à l'espace, à la forêt [...]. Et c'est ainsi que survint en mon imagination l'idée d'une représentation spéciale, dont les lois que j'entrevoyais seraient exactement à l'opposé des lois théâtrales [...]. II ne s'agissait pas de réunir dans une des vastes salles un grand nombre de personnes et de jouer une tragédie devant elles. Non, la tragédie se déroulerait dans les décors séculaires que m'offrait l'abbaye. Les spectateurs réduits à un minimum la suivraient en silence et, protégés par la nuit, la surprendraient comme des indiscrets ${ }^{13}$.

Ce n'est rien moins qu'à une nouvelle conception du théâtre - et comme nous le verrons à une expérience de théâtre total - que nous invite Georgette Leblanc - qui en réalisa seule la mise en scène - un théâtre débarrassé des contraintes de la scène, où le spectateur est invité à prendre part à ce qui se joue devant ses yeux, comme en témoignent les costumes distribués à ceux qui le souhaitent en début de soirée ${ }^{14}$.

\section{Acte II : Saint-Wandrille, juillet 1909}

Macbeth est donc mis en scène pour une représentation unique le 27 août 190915, devant une soixantaine de spectateurs, parmi lesquels la princesse Murat, Adophe Brisson, Yvonne Sarcey, Jacques Hébertot, Francis de Croisset et bien entendu Gaston Calmette, le directeur

10 lbid. 11 lbid. 12 La traduction paraît en 1910 chez Charpentier et Fasquelle, sous le titre la Tragédie de Macbeth. Il convient d'ailleurs de parler plutôt d'une adaptation tant les écarts avec la version originale sont nombreux : coupes dans I'acte III, refonte complète du dernier acte, etc. $13 \mathrm{G}$. Leblanc, op. cit., cité par M. Benoît-Jeannin, op. cit., p. 275. 14 D'après Georgette Leblanc, Maeterlinck aurait lui-même été déguisé en soldat écossais, Souvenirs, op. cit., p. 240. 15 La distribution comprend - outre Georgette Leblanc dans le rôle de Lady Macbeth - Séverin-Mars (Macbeth), René Maupré et Mme Cassini de I'Odéon, Andreyor du théâtre de la Renaissance et Schütz du Théâtre Sarah-Bernhardt. 
du Figaro qui a rendu la chose possible grâce à la souscription lancée dans son journal16. Les spectateurs, répartis par groupes de dix, sont dispersés sur l'ensemble du site, et guidés par des figurants muets vers les principales scènes, si bien que la plupart d'entre eux pourront se prévaloir de n'avoir pas vu la même pièce ${ }^{17}$. Les costumes dessinés par Georgette Leblanc sont inspirés de la Tapisserie de Bayeux et la musique composée par un certain Charles Bordes I'a été d'après un manuscrit du XIII' siècle conservé à Oxford ${ }^{18}$. Cinquante comédiens et figurants animent une tragédie qui se déroule sur l'ensemble du site, illuminé de braseros. Cela tient du spectacle "son et lumières" tant on mobilise les paysans de la région pour interpréter les figurants (bien que les conditions d'audition aient parfois été problématiques si l'on en croit un compte rendu de Guillot de Saix ${ }^{19}$ ), du péplum (I'arrivée à cheval du roi Duncan et de sa suite devant la porte de l'abbaye a, semble-t-il, marqué les esprits), et pour tout dire d'un théâtre total qui fait littéralement exploser les murs de la salle20.

L'émotion procurée au spectateur devait être " plus près de la vie que du théâtre », conduite par la force du lieu qui rend les conditions de la tragédie particulièrement difficiles : Une intonation fausse, une scène dite sans vérité étaient impossibles. Le sens de l'abbaye s'imposait mystérieusement. Ses proportions géantes interdisaient l'erreur, en la grossissant monstrueusement comme l'objectif de la caméra. Les échos multiples nécessitaient une diction parfaite et interdisaient la moindre affectation ${ }^{21}$.

Le jeu se trouve donc modifié et comme renouvelé par la structure même du lieu, à l'image de ce qui se passe devant une caméra. L'évocation du cinéma ne doit toutefois pas nous induire en erreur ici. Il ne s'agit pas de prescience, mais plutôt de la claire perception de ce que le cinéma peut malencontreusement ajouter à la performance de l'acteur, en l'absence d'une parole audible. Mais au cinéma22, comme à Saint-Wandrille en 1909, I'acteur doit reconsidérer son jeu et s'inscrire dans ce que les observateurs de l'époque appellent " l'atmosphère » du lieu. À propos de la représentation de Pelléas et Mélisande qui eut lieu le 28 août $1910^{23}$

16 Le montant minimum était fixé à 200 francs or, ce qui représente une somme assez considérable. 17 On retrouvera semblable circulation du public lors de la représentation de Pelléas et Mélisande un an plus tard. 18 Ces renseignements proviennent de M. Benoît-Jeannin, op. cit. 19 Ce compte rendu concerne toutefois la représentation de Pelléas et Mélisande en 1915 : " Il faut se déplacer à chaque tableau et, tout en suivant les personnages, on doit faire attention où l'on met le pied. L'acoustique n'est pas toujours favorable et la divine illusion s'enfuit ", Guillot de Saix, "Pelléas et Mélisande à l'abbaye de Saint-Wandrille », s. n., 29 août 1915. 20 Georgette Leblanc organisa d'ailleurs sa mise en scène en travaillant d'après un plan de l'abbaye qu'elle avait fait établir à cette fin. 21 G. Leblanc, op. cit., p. 244. Nous soulignons. 22 Cf. Id., "Propos sur le cinéma », Mercure de France, 16 novembre 1919, p. $275-290$. 23 Les principaux rôles y étaient tenus par Georgette Leblanc (Mélisande), René Maupré (Pelléas), Séverin-Mars (Arkel), Jeanne Even (Geneviève). La pièce était accompagnée par la musique de Gabriel Fauré, interprétée par un orchestre dissimulé derrière les murs de la l'abbaye. On compte parmi les vingt-cinq spectateurs Lucien Guitry, Réjane et le jeune Marcel L'Herbier. 
dans les mêmes conditions que Macbeth, Georges Bourdon écrit : « [...] L'éblouissement de Pelléas et Mélisande est sans pareil. Jamais ne fut à ce point restituée, avec son âme propre dans son rythme intime, avec ce qu'on appelle son atmosphère, une œuvre littéraire. Jamais le génie d'un créateur n'eut à son service, au premier degré, le génie d'une interprète ${ }^{24}$. Il semble que Georgette Leblanc ait éminemment désiré monter un Hamlet et une Princesse Maleine en 1912 et 1913, mais elle ne put trouver à nouveau le financement nécessaire à une telle entreprise. Consciente du renouvellement possible de l'expression théâtrale dans un tel contexte, elle aurait souhaité installer une école à Saint-Wandrille, destinée à accueillir des élèves qui eussent contribué aux nouvelles mises en scène de la comédienne. Si l'on en croit ses Souvenirs, le projet était grandiose :

J'aurais voulu constituer à Saint-Wandrille un Bayreuth français. C'eût été la fortune pour le pays et un centre d'art incomparable. Outre les drames de Maeterlinck qui presque tous s'adaptaient à l'abbaye, je voulais faire revivre là, à leur place, les Mystères du Moyen Âge enveloppés de chants grégoriens par les chœurs de Saint-Gervais. Ce que mes spectateurs ont trouvé unique et merveilleux n'était pour moi qu'un coup d'essai25.

Par chance, ces « coups d'essai » devaient être partiellement enregistrés par la caméra.

\section{Acte III : Neuilly, hiver 1915}

Les 29 et 30 novembre 1915 ont lieu au Pavillon des Muses, 96 boulevard Maillot à Neuilly les premières projections publiques de la captation cinématographique de Macbeth et de Pelléas et Mélisande. Le Figaro atteste le rôle de Gustave Labruyère, présenté comme un « imprésario ", auteur de l'adaptation cinématographique des deux pièces ${ }^{26}$. Les représentations ont lieu - guerre oblige - au profit de l'ÆEuvre fraternelle des artistes et des œuvres d'assistance belges $^{27}$. Et si l'on en croit le Figaro du 30 novembre, les cent-cinquante places de la petite salle ont toutes trouvé preneur.

Ces captations cinématographiques - fort écourtées au demeurant puisque le Pelléas de 1910 durait selon les témoins près de trois heures 28 - n'ont pas été réalisées lors des premières

24 G. Bourdon, « Dans le palais de Pelléas et Mélisande », le Figaro, 31 août 1910. Le dithyrambe est néanmoins à considérer avec quelque précaution : le Figaro était en effet le principal soutien financier de la représentation. 25 G. Leblanc, op. cit., p. 243. 26 Le Figaro, 19 novembre 1915. 27 Rappelons que Maurice Maeterlinck était de nationalité belge. 28 La version conservée par la Cinémathèque de Toulouse ne dépasse pas vingt minutes. 
représentations des deux pièces, mais à l'occasion d'une reprise qui eut lieu pendant l'été 191529, dans la propriété de Saint-Wandrille. Peut-être est-ce pour les besoins du cinématographe que certaines scènes furent alors éclairées à l'électricité ${ }^{30}$. Les costumes des représentations de 1909 et 1910 furent probablement réutilisés, une souscription - toujours soutenue par le Figaro - étant à nouveau ouverte pour l'organisation du spectacle; cette dernière réunit personnalités du monde (le baron Empain, la princesse de Polignac, la princesse de Monaco, le baron Edmond de Rotschild...) et de la politique - plus présents qu'en 1909-1910 (le président Poincaré, Viviani, président du Conseil, Delcassé, ministre des Affaires étrangères, Millerand, ministre de la guerre, les présidents de la Chambre et du Sénat figurent parmi les souscripteurs). Il nous est précisé que les bénéfices en seraient versés à la Fraternelle des artistes. La part prise par les politiques à l'organisation du spectacle (la presse ne mentionne pas qu'ils se fussent déplacés) laisse penser que l'entreprise était considérée comme un emblème de la vivacité de l'art français menacé par la guerre.

Au demeurant, Georgette Leblanc ne mentionne qu'à peine cette reprise dans ses Souvenirs, sinon pour indiquer qu'elle donna effectivement lieu à une captation cinématographique. Ce silence ne laisse pas d'étonner, tant la comédienne montra par la suite son intérêt pour le cinéma et son désir d'y figurer à nouveau ${ }^{31}$. Peut-être considéra-t-elle le résultat comme décevant, en-deçà des espoirs et des attentes qu'elle avait placés dans sa mise en scène, y décelant un écart entre son projet et la captation cinématographique ${ }^{32}$, peut-être fut-elle également désappointée par le peu d'échos des projections de Neuilly, au regard des représentations de Saint-Wandrille. Il est vrai que le cadre choisi par Gustave Labruyère ne laisse guère de place au dynamisme de l'espace théâtral voulu par Georgette Leblanc. Au contraire, si l'on s'arrête un instant sur Macbeth, le film semble bien statique pour une production du milieu des années 1910. Si les scènes d'extérieur donnent un aperçu des représentations originelles, les plans d'intérieur réalisés principalement dans le réfectoire (le banquet offert au roi Duncan par Macbeth) pâtissent d'une exécution sommaire. Quant au jeu des comédiens, il ne reste pas grand chose de la sobriété promue et revendiquée par Georgette Leblanc. En tout état de cause, nous ignorons, en l'état actuel de nos recherches, si les deux films furent distribués par la suite.

29 Le 19 août pour Macbeth, le 22 pour Pelléas. 30 Le Flambeau, 28 août 1915. 31 Elle envisagea de tourner une Marie-Magdeleine, d'après la pièce de Maeterlinck, ainsi qu'une Madone du secret et une adaptation de l'Oiseau bleu qui fut bien réalisée aux États-Unis en 1918 par Maurice Tourneur, mais sans Georgette Leblanc. Enfin, Marcel L'Herbier écrivit un scénario pour elle, le Torrent, dans lequel il lui fut impossible de jouer en raison d'une maladie. 32 La phrase citée plus haut sur le grossissement des erreurs induit par la présence de la caméra prendrait alors tout son sens. 


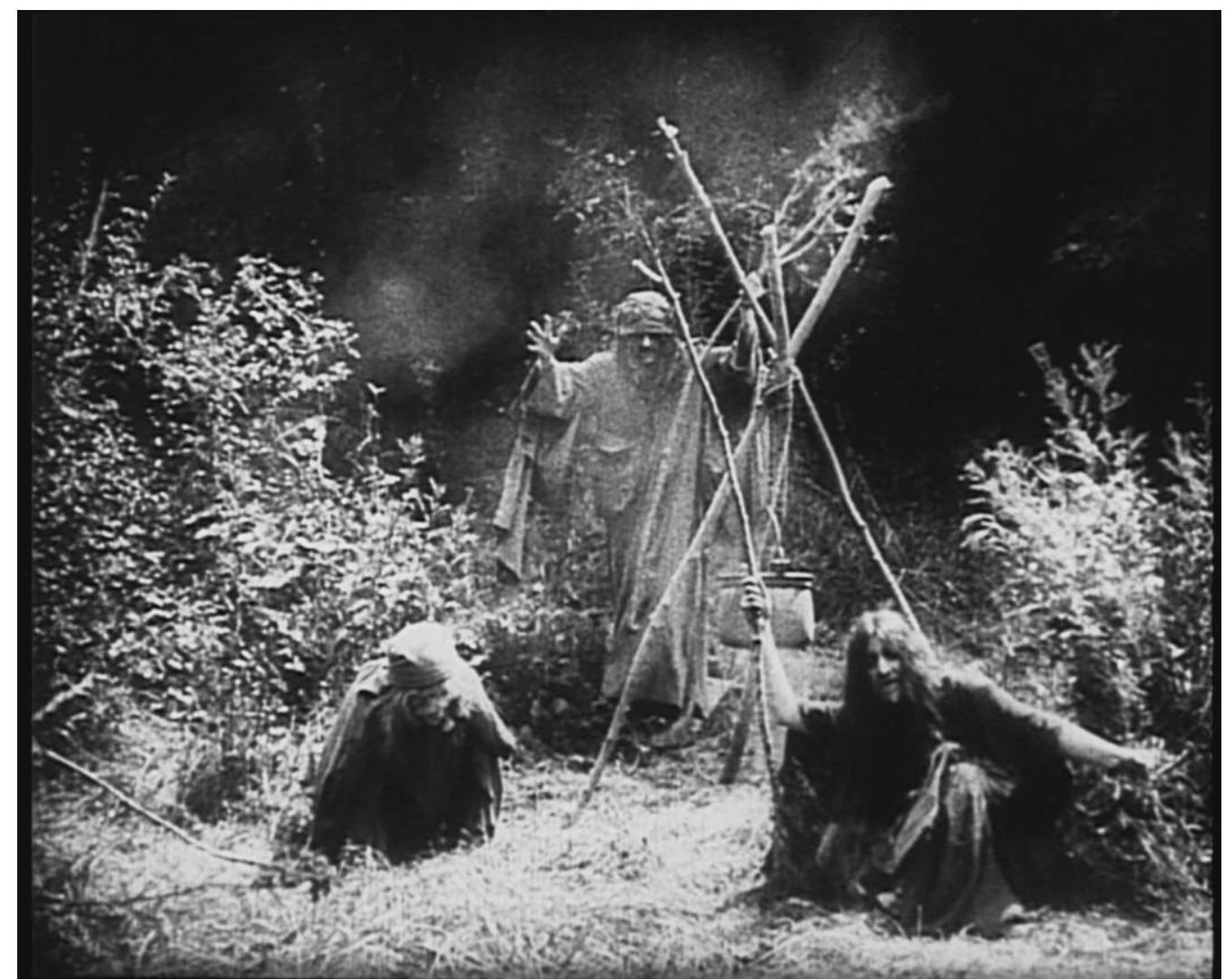

Macbeth, Georgette Leblanc, 1915, coll. La Cinémathèque de Toulouse.

\section{Acte IV : Retour à Saint-Wandrille, été 1908}

Le couple Maeterlinck recevait beaucoup à Saint-Wandrille, et ce dès la première année. À l'été 1908, Maurice Maeterlinck y invite l'homme qui doit mettre en scène la première de I'Oiseau bleu à Moscou33 : il s'agit de Constantin Stanislavski qui suit le travail du poète depuis 1904. Maeterlinck ayant l'habitude de s'isoler régulièrement pour écrire, Stanislavski noue des relations amicales avec Georgette Leblanc et évoque une mise en scène de Pelléas dans les lieux-mêmes où la pièce sera représentée deux ans plus tard:

33 Elle aura lieu le 30 septembre 1908 au Théâtre d'art de Moscou, en présence de Georgette Leblanc ; Maeterlinck, lui, séjourne alors aux « Abeilles». 
Dans la journée, tandis que le poète travaillait, je me promenais avec Mme Maeterlinck à travers I'abbaye en rêvant de monter là, en plein air, Aglavaine et Sélisette ou Pelléas et Mélisande.

En divers endroits de l'abbaye, nous traversâmes des coins pittoresques qui semblaient faits tout exprès pour monter les pièces de Maeterlinck : ici, caché dans la verdure, le puits médiéval où Pelléas rencontre Mélisande, ailleurs l'entrée d'un souterrain pour la scène entre Pelléas et Golaud, etc.

Nous projetâmes d'organiser une représentation où les spectateurs se déplaceraient en même temps que les acteurs d'un endroit de l'abbaye à un autre pour pouvoir regarder la pièce. Si je ne me trompe, nos rêves se réalisèrent par la suite grâce à Mme Georgette Leblanc-

Cette dernière reconnaîtra d'ailleurs indirectement le rôle décisif joué par Stanislavski dans la genèse de cette aventure théâtrale, en évoquant le projet d'école « libre » du théâtre qu'elle aurait désiré créer à Saint-Wandrille, sur les conseils de son ami russe : " Les cours, les leçons, les répétitions ne peuvent apporter aux artistes ce que donne une continuité d'atmosphère, la vie de l'esprit dans un cadre de beauté. Je vous envie cette abbaye ", faitelle dire à Stanislavski dans ses Souvenirs ${ }^{35}$. Bien entendu, cette contribution minuscule du grand rénovateur russe de la mise en scène théâtrale à la mise en espace de Pelléas n'ôte rien aux qualités ni à l'énergie déployées par Georgette Leblanc pour la réalisation de ce rêve. Et d'ailleurs, il n'est pas question de Macbeth dans les souvenirs de Stanislavski. II me plaît toutefois de penser que l'ombre lointaine de Stanislavski plane sur les films conservés à la Cinémathèque de Toulouse, qu'à un Shakespeare 1900 se mêle l'avant-garde théâtrale de l'époque, que l'on ait rêvé de Bayreuth à Saint-Wandrille, qu'un siècle englouti, enfin, puisse ressurgir sur les écrans. Le Macbeth et le Pelléas de Georgette Leblanc sont pareils aux figures du salon de la Princesse de Guermantes dans le Temps retrouvé : ils viennent à nous comme les fantômes d'un monde perdu³.

34 C. Stanislavski, Ma vie dans l'art, Lausanne, L'Âge d'homme, 1999, p. 403.35 G. Leblanc, op. cit., p. 244. 36 Cette étude est un préalable à une restauration complète des deux films. Nous poursuivons notre enquête auprès des cinémathèques du monde entier, et nous attendons une réponse de la Cinémathèque de Bucarest qui a peut-être conservé les nitrates d'origine. Par ailleurs, nous espérons être en mesure de livrer aux lecteurs une analyse des deux films à la lumière des faits exposés ici. 